\title{
NÚMEROS DE CASOS CONFIRMADOS DE SÍFILIS EM GESTANTES NO BRASIL ENTRE 2009 A 2013
}

\section{ARTIGO ORIGINAL}

BARROS, Yara Lorrane Souza de ${ }^{1}$, FECURY, Amanda Alves², OLIVEIRA, Euzébio de $^{3}$, DENDASCK, Carla Viana ${ }^{4}$, ARAÚJO, Maria Helena Mendonça de ${ }^{5}$, SOUZA, Keulle Oliveira da ${ }^{6}$, DIAS, Claudio Alberto Gellis de Mattos ${ }^{7}$

BARROS, Yara Lorrane Souza de. Et al. Números de casos confirmados de sífilis em gestantes no Brasil entre 2009 a 2013. Revista Científica Multidisciplinar Núcleo do Conhecimento. Ano 05, Ed. 11, Vol. 25, pp. 53-61. Novembro de 2020. ISSN: 2448-0959, Link de acesso:

https://www.nucleodoconhecimento.com.br/saude/sifilis-em-gestantes, DOI: 10.32749/nucleodoconhecimento.com.br/saude/sifilis-em-gestantes

\section{RESUMO}

Sífilis é uma doença bacteriana transmitida sexualmente da gestante portadora de sífilis para o feto. O diagnóstico da sífilis em gestante é confirmado através do exame VDRL. O objetivo deste trabalho é mostrar os números de casos confirmados de sífilis em gestantes no Brasil, quanto ano de diagnostico, faixa etária, regiões do Brasil, zona residencial, tipo de teste, classificação e evolução entre 2009 a 2013. Foram utilizados dados secundários retirados do departamento de informática do SUS - DATASUS (http://datasus.saude.gov.br). O maior número de exames

\footnotetext{
${ }^{1}$ Técnica em Mineração, egressa do Instituto Federal do Amapá (IFAP).

2 Biomédica, Doutora em Doenças Tropicais, Professora e pesquisadora do Curso de Medicina da Universidade Federal do Amapá (UNIFAP).

${ }^{3}$ Biólogo, Doutor em Doenças Topicais, Professor e pesquisador do Curso de Educação Física da Universidade Federal do Pará (UFPA).

${ }^{4}$ Teóloga, Doutora em Psicanálise, pesquisadora do Centro de Pesquisa e Estudos Avançados- CEPA.

${ }^{5}$ Médica, Professora e pesquisadora do Curso de Medicina da Universidade Federal do Amapá (UNIFAP).

${ }^{6}$ Socióloga, Mestranda em Estudos Antrópicos na Amazônia, Integrante do Grupo de Pesquisa "Laboratório de Educação, Meio Ambiente e Saúde" (LEMAS/UFPA).

${ }^{7}$ Biólogo, Doutor em Teoria e Pesquisa do Comportamento, Professor e pesquisador do Programa de PósGraduação em Educação Profissional e Tecnológica (PROFEPT), Instituto Federal do Amapá (IFAP).
}

RC: 66576

Disponível em: https://www.nucleodoconhecimento.com.br/saude/sifilis-em-gestantes 
possibilita um melhor tratamento e diminuição do número de casos. Entretanto, a ausência de cuidado preventivo durante o relacionamento sexual induz ao aumento de casos, principalmente entre mulheres em idade reprodutiva. Áreas com maior população apresentam maiores números de casos. A variação alta de parceiros nestas áreas impulsiona o aumento de contaminação. O tipo de teste influencia nos números. Testes menos específicos, mais gerais, localizam anticorpos específicos e não específicos para a bactéria. Isso pode alterar os números reais. $O$ cuidado feminino com a saúde faz com quer o diagnóstico seja precoce, facilitando o tratamento. A sífilis não é responsável direta pelo maior número de mortalidade em gestantes.

Palavras-chaves: Epidemiologia, sífilis, gestante.

\section{INTRODUÇÃO}

Sífilis é uma doença bacteriana que afeta o corpo inteiro, transmitida sexualmente; por doação sanguínea; pelo contato direto com o sangue contaminado; e da gestante portadora de sífilis para o feto. A bactéria causadora da doença é a Treponema Pallidum (CARVALHO et al., 2014, JUNIOR et al., 2009; ROCHA et al., 2020).

Os sintomas da sífilis se manifestam em três fases. Na primeira fase podem aparecer manchas vermelhas pelo corpo, feridas geralmente nas partes genitais. $\mathrm{Na}$ segunda fase é possível que a doença fique adormecida por um período de até oito meses, quando a bactéria pode distribuir-se afetando a pele e os órgãos. Na terceira fase é comum a aparição de inflamações nos tecidos do corpo, como os ossos, músculos e fígado (AVELLEIRA et al., 2006; BRETAS et al., 2008).

A doença precisa ser diagnosticada para que o paciente possa fazer o tratamento adequado, prevenindo a transmissão da doença através do uso de camisinha masculina/feminina. $O$ tratamento mais eficaz é a droga penicilina benzatina. Até o ano de 2015 não houve registro de nenhum caso de resistência a ele. Outras drogas

$\mathrm{RC}: 66576$

Disponível em: https://www.nucleodoconhecimento.com.br/saude/sifilis-em-gestantes 
são utilizadas no tratamento da sífilis, mas a que apresenta maior eficiência no tratamento/cura é este tipo de penicilina (KALININ et al., 2015; BRASIL, 2015).

O diagnóstico da sífilis em gestante é confirmado através do exame VDRL (Venereal Disease Research Laboratory = Pesquisa Laboratorial de Doença venérea) durante o pré-natal. A descoberta da doença e o tratamento da gestante durante o pré-natal com penicilina previnem à sífilis congênita (SUTO et al., 2016; MESQUITA et al., 2012).

O número de casos de sífilis em gestantes no Brasil foi 21382, em 2012 e 2013 (BRASIL 2015).

O número de casos de sífilis em gestantes na região Nordeste foi 4.433, em 2012 e 2013. No mesmo período o número de casos de sífilis em gestantes na região Sudeste foi de 10.052; na região Sul foi 2795; na região Centro-Oeste foi 1961; e na região Norte foi 214 (BRASIL, 2015).

O número de casos sífilis em gestantes no Amapá foi 176, em 2012 e 2013 (BRASIL 2015).

\section{OBJETIVO}

O objetivo deste artigo é mostrar os números de casos confirmados de sífilis em gestantes no Brasil, quanto ano de diagnostico, faixa etária, regiões do Brasil, zona residencial, tipo de teste, classificação e evolução entre 2009 a 2013.

\section{MÉTODO}

Dados retirados do departamento de informática do SUS - DATASUS (http://datasus.saude.gov.br) seguindo as seguintes etapas: primeiro selecionou-se a Aba "acesso à informação" em seguida a opção "informações de saúde (TABNET)" logo depois a opção "epidemiológicas e morbidade". Clicou-se na opção "Doenças e Agravos de Notificação De 2007 em diante (SINAN)" e em seguida "Sífilis em

RC: 66576

Disponível em: https://www.nucleodoconhecimento.com.br/saude/sifilis-em-gestantes 
Gestante", e na aba "abrangência geográfica" selecionou "Brasil por Região, UF e Município". As próximas etapas foram: A) na linha selecionou-se "Casos confirmados segundo Ano de Diagnóstico", na coluna "não ativo" e em conteúdo "casos confirmados". Para esta opção e para as demais a seguir os dados foram coletados no período de 2009 a 2013. B) na linha selecionou-se "Casos confirmados segundo Região de notificação", na coluna "não ativo" e em conteúdo "casos confirmados". C) na linha selecionou-se "Casos confirmados segundo Faixa Etária", na coluna "não ativo" e em conteúdo "casos confirmados". D) na linha selecionou-se "Casos confirmados segundo Evolução", na coluna "não ativo" e em conteúdo "casos confirmados". E) na linha selecionou-se "Casos confirmados segundo Classificação clínica", na coluna "não ativo" e em conteúdo "casos confirmados". F) na linha selecionou-se "Casos confirmados segundo Teste não Trep", na coluna "não ativo" e em conteúdo "casos confirmados". G) na linha selecionou-se "Casos confirmados segundo Teste Trep", na coluna "não ativo", em conteúdo "casos confirmados". H) na linha selecionar "Casos confirmados por Zona Residência segundo Ano de Diagnóstico", na coluna selecionar "Zona Residencial" e em conteúdo "casos confirmados". A compilação dos dados foi feita dentro do aplicativo Excel, componente do pacote Office da Microsoft Corporation. A pesquisa bibliográfica foi realizada em artigos científicos, utilizando-se para busca computadores do laboratório de informática do Instituto Federal de Educação, Ciência e Tecnologia do Amapá, Campus Macapá, situado na: Rodovia BR 210 KM 3, s/n - Bairro Brasil Novo. CEP: 68.909-398, Macapá, Amapá, Brasil.

\section{RESULTADOS}

A figura 1 mostra o número de casos confirmados de sífilis em gestantes por ano no Brasil entre os anos de 2009 a 2013. Os dados mostram um crescimento no número de casos confirmados de 2009 a 2012. Este número caiu pela metade no ano de 2013 quando comparado ao ano anterior.

$\mathrm{RC}: 66576$

Disponível em: https://www.nucleodoconhecimento.com.br/saude/sifilis-em-gestantes 
Figura 1 Número de casos confirmados de sífilis em gestantes por ano no Brasil entre os anos de 2009 a 2013.

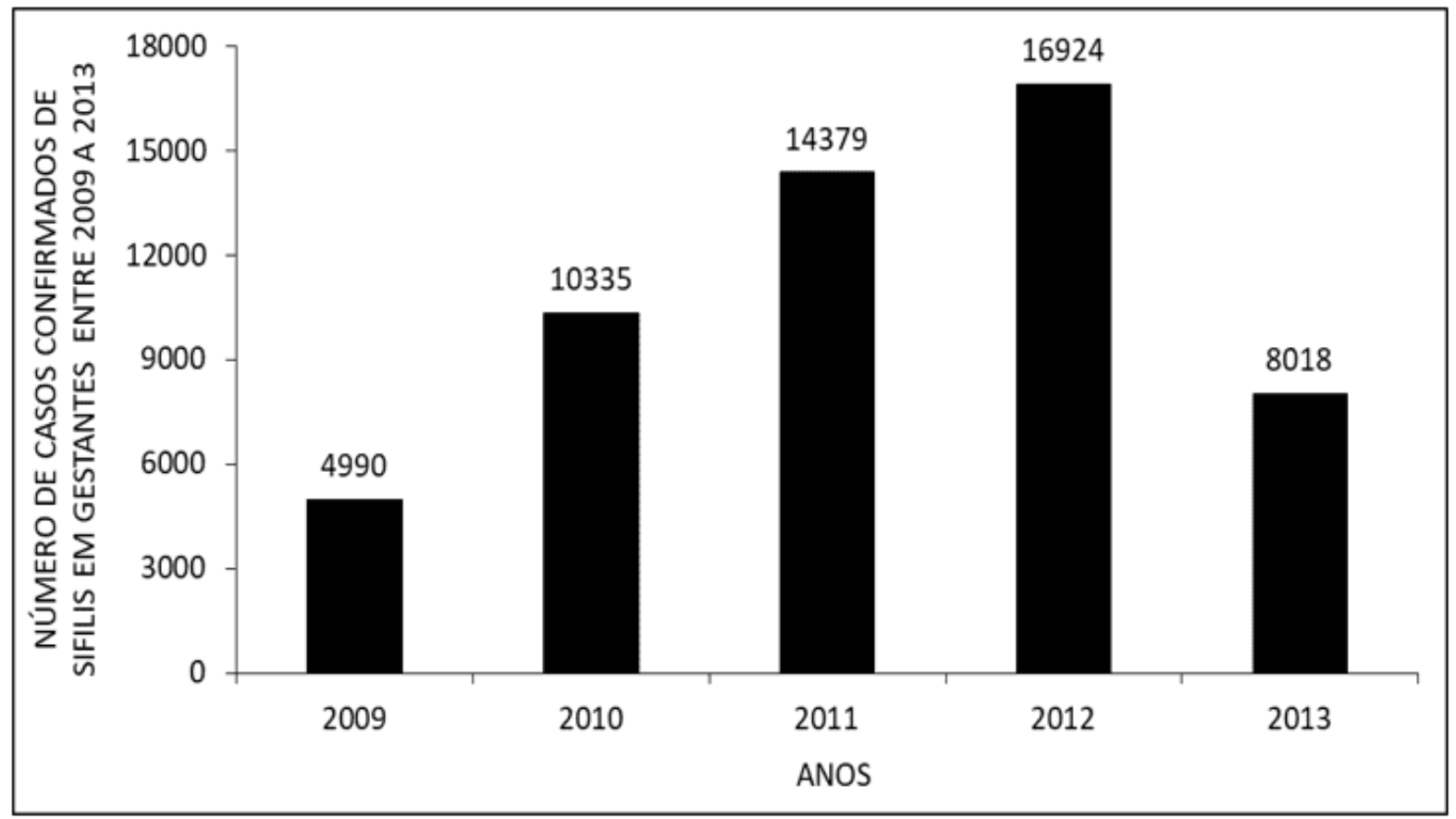

A figura 2 mostra o número de casos confirmados de sífilis em gestantes por faixa etária no Brasil entre os anos de 2009 a 2013. Entre 10 e 39 anos de idade houve um crescimento. Este número diminui entre 40 e 64 anos de idade.

RC: 66576

Disponível em: https://www.nucleodoconhecimento.com.br/saude/sifilis-em-gestantes 
Figura 2 Número de casos confirmados de sífilis em gestantes por faixa etária no Brasil entre os anos de 2009 a 2013.

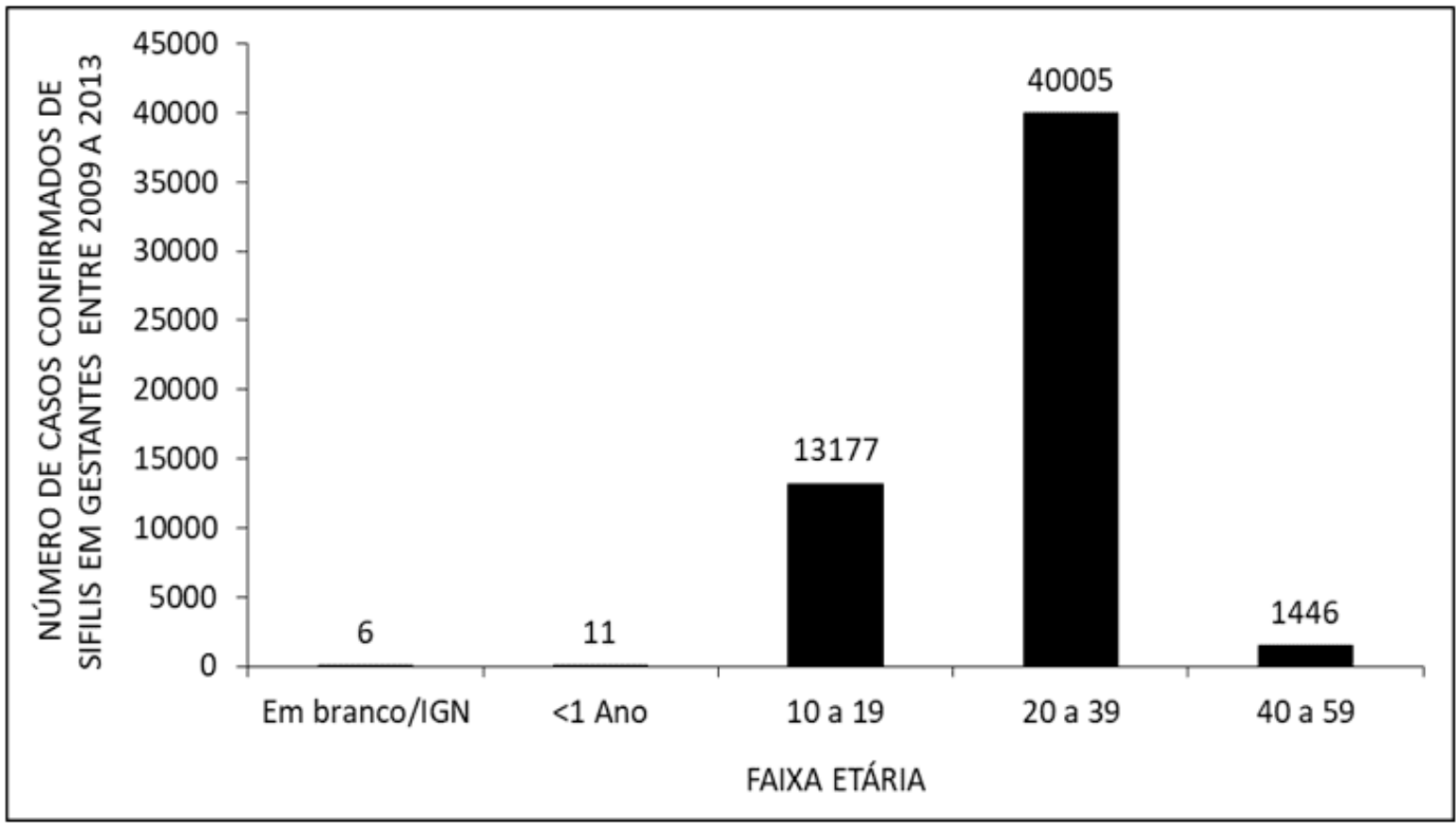

A figura 3 mostra o número de casos confirmados de sífilis em gestantes por região no Brasil entre os anos de 2009 a 2013. A região sudeste possui o maior número de casos confirmados, seguida respectivamente pelas regiões nordeste, norte, sul e centro-oeste.

RC: 66576

Disponível em: https://www.nucleodoconhecimento.com.br/saude/sifilis-em-gestantes 
Figura 3 Número de casos confirmados de sífilis em gestantes por região do país no Brasil entre os anos de 2009 a 2013.

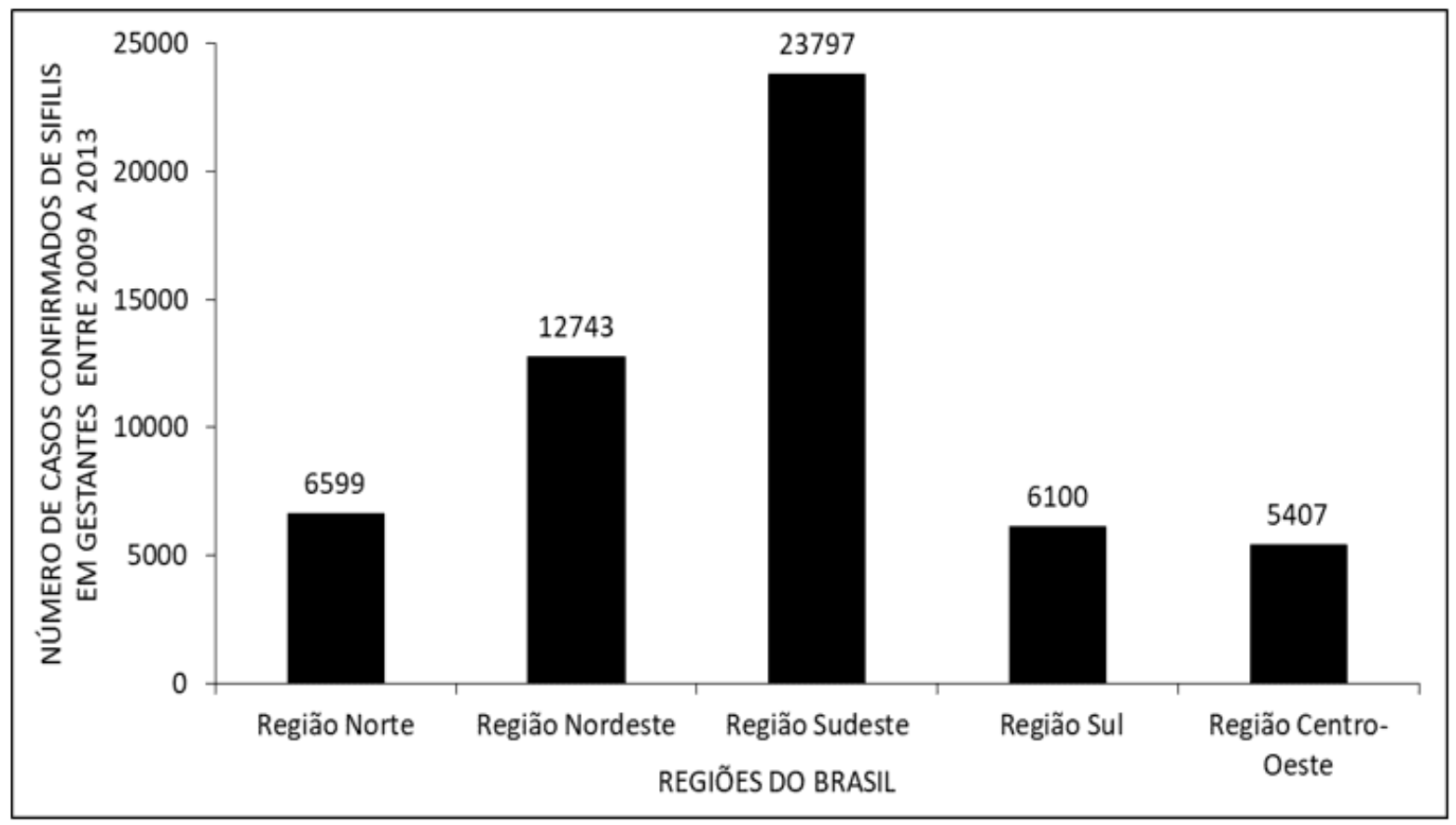

A figura 4 mostra o número de casos confirmados de sífilis em gestantes por zona de residência no Brasil entre os anos de 2009 a 2013. O número na zona urbana é aproximadamente 8 vezes maior que na zona rural. $O$ menor número de casos por zona de residência é respectivamente periurbana e lgn/Branco.

RC: 66576

Disponível em: https://www.nucleodoconhecimento.com.br/saude/sifilis-em-gestantes 
Figura 4 Número de casos confirmados de sífilis em gestantes de acordo com o tipo de zona de residência no Brasil entre os anos de 2009 a 2013.

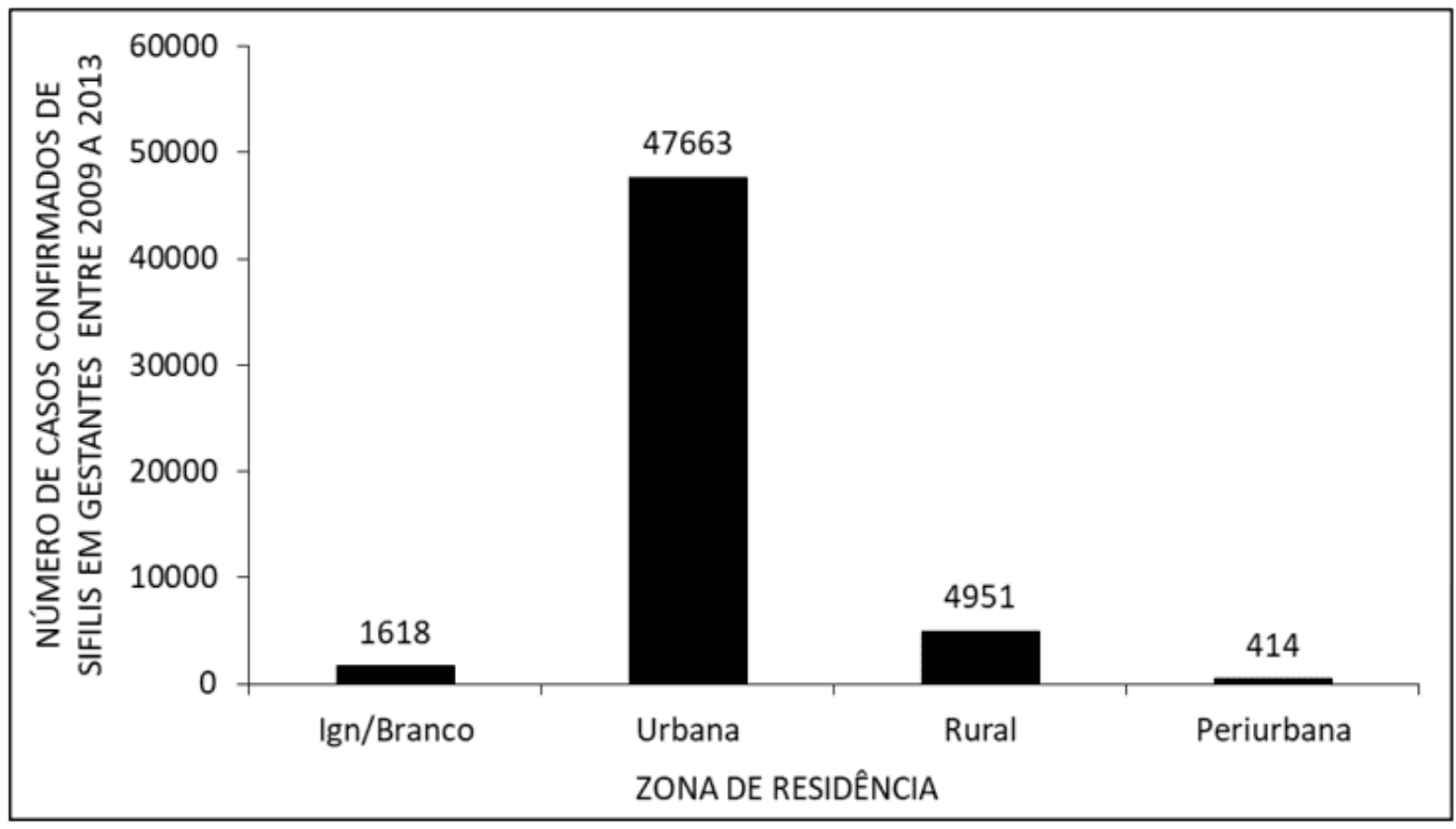

A figura 5 mostra o número de casos confirmados de sífilis em gestantes por tipo de teste realizado no Brasil entre os anos de 2009 a 2013. O número de casos de testes realizados Ign/brancos por teste TREP é quadro vezes maior que testes NÃO TREP. O número de casos reativos por testes NÃO TREP é quase o dobro de casos reativos por testes TREP. O número de casos não reativos entre testes NÃO TREP e TREP são aproximados. O número de casos não realizados de testes TREP é nove vezes maior que o número de casos não realizados por testes NÃO TREP.

RC: 66576

Disponível em: https://www.nucleodoconhecimento.com.br/saude/sifilis-em-gestantes 
Figura 5 Número de casos confirmados de sífilis em gestantes de acordo com o tipo de teste realizado no Brasil entre os anos de 2009 a 2013.

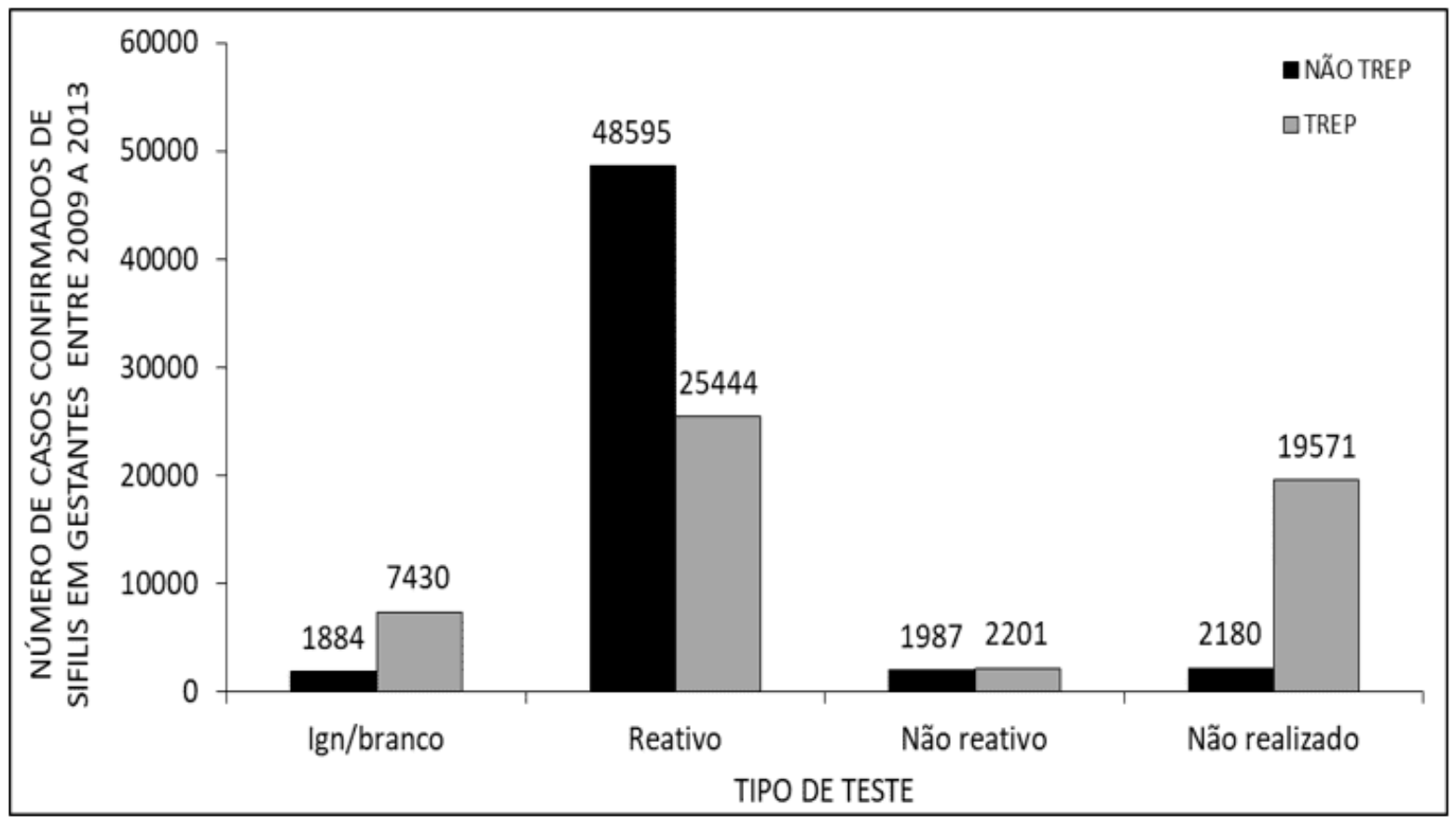

A figura 6 mostra o número de casos confirmados de sífilis em gestantes de acordo com a classificação no Brasil entre os anos de 2009 a 2013. A classificação primaria possui o maior número de casos, seguida respectivamente por lgn/Branco e latente. As classificações, secundária e terciária, possuem o menor número de casos confirmados.

RC: 66576

Disponível em: https://www.nucleodoconhecimento.com.br/saude/sifilis-em-gestantes 
Figura 6 Número de casos confirmados de sífilis em gestantes de acordo com a classificação no Brasil entre os anos de 2009 a 2013.

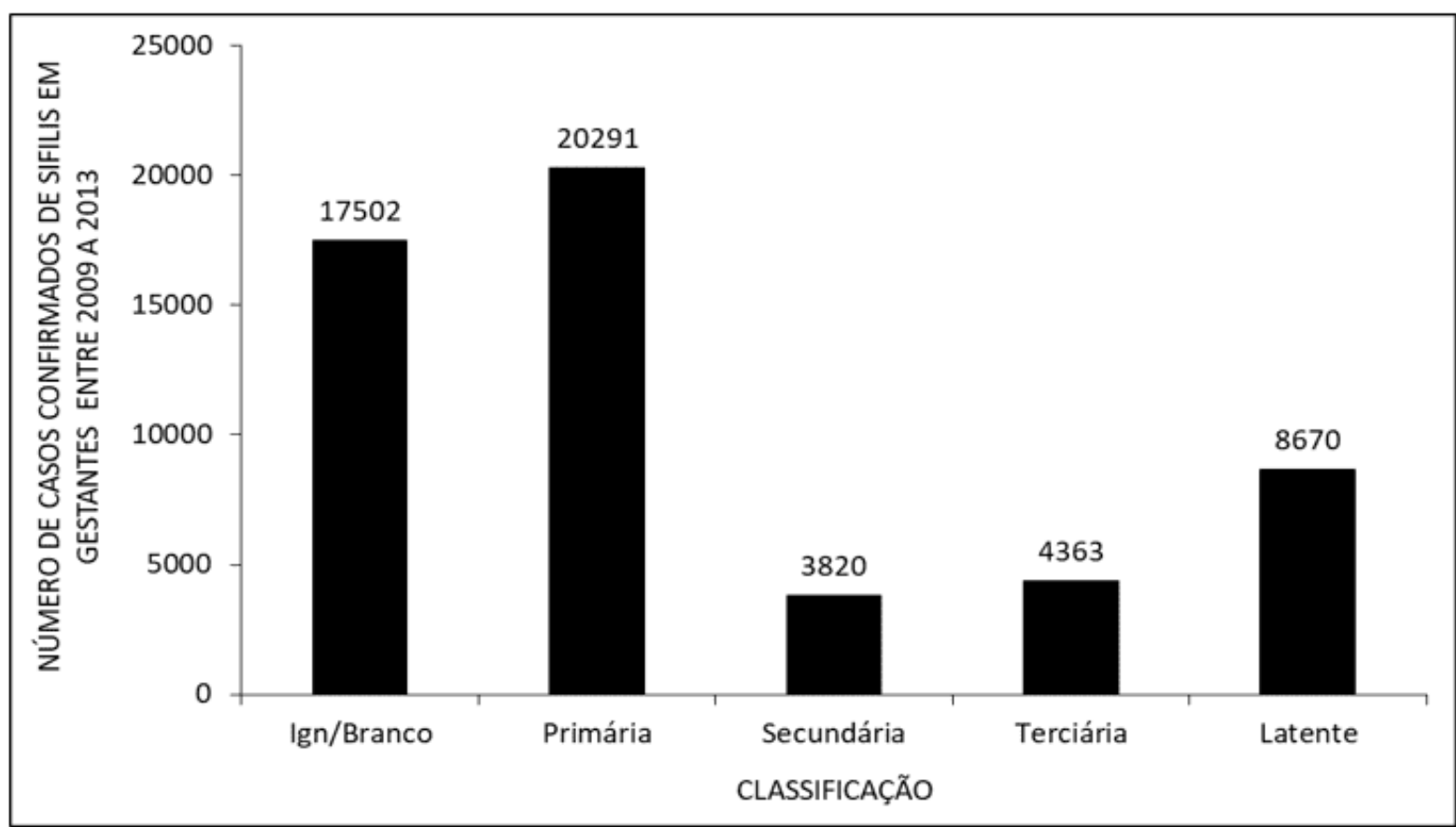

A figura 7 mostra o número de casos confirmados de sífilis em gestantes de acordo com a evolução no Brasil entre os anos de 2009 a 2013. Os dados mostram que o número de óbitos de gestantes que adquiriram sífilis neste período foi o dobro do número de curas. O maior número de óbitos ocorreu por outras causas. $\mathrm{O}$ menor número de óbitos do período apareceu como em investigação.

RC: 66576

Disponível em: https://www.nucleodoconhecimento.com.br/saude/sifilis-em-gestantes 
Figura 7 Número de casos confirmados de sífilis em gestantes de acordo com a evolução no Brasil entre os anos de 2009 a 2013.

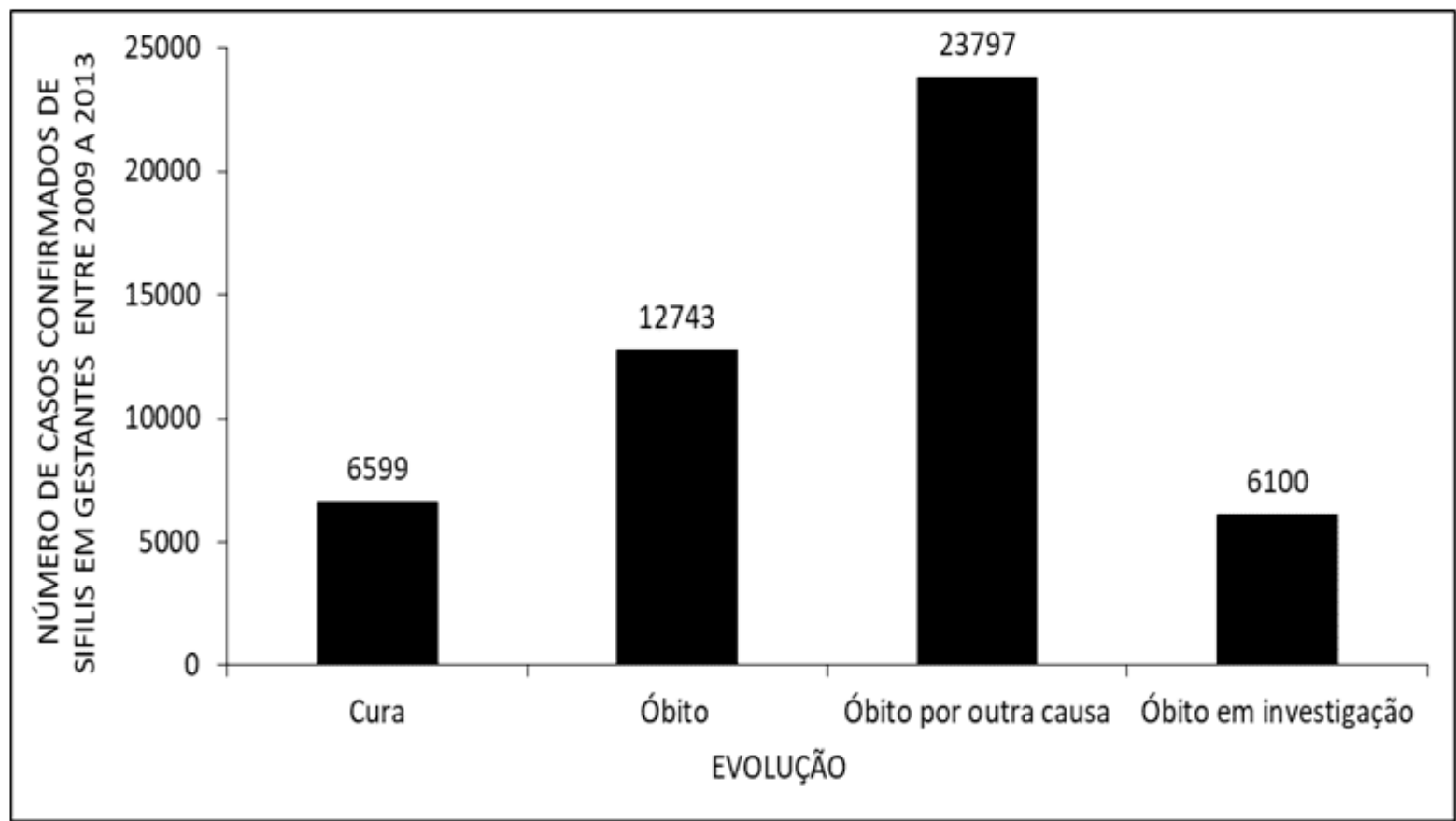

\section{DISCUSSÃO}

Houve um crescimento no número de casos confirmados de sífilis em gestante entre os anos de 2009 a 2012. Entre 2011 e 2013 houve um aumento do número de exames, de 31,5 mil para 1,7 milhão, o que parece ter possibilitado o tratamento da paciente com sífilis. Este panorama pode ter influenciado na queda de casos confirmados em gestantes no ano de 2013 (BRASIL, 2013; 2017).

Os dados mostram casos de sífilis em gestantes aumentados na faixa de 10 a 39 anos e sua diminuição entre 40 e 64 anos (figura 2), corroborados pela literatura. $\mathrm{Na}$ faixa etária entre 20-34 anos, as mulheres estão no auge da sua fase reprodutiva. Parece existir um índice maior de gestantes com sífilis nesta faixa etária, possivelmente explicado pela prática do sexo sem proteção (COSTA et al, 2012).

RC: 66576

Disponível em: https://www.nucleodoconhecimento.com.br/saude/sifilis-em-gestantes 
O Sudeste é a região brasileira que possui o maior número de casos de sífilis em gestantes no período pesquisado. Este dado pode ser explicado pelo fato de o Sudeste concentrar a maior parte da população brasileira e aproximadamente metade da população admite não usar camisinha em relações sexuais (BRASIL, 2011; IBGE, 2010).

A zona urbana do Brasil apresentou, no período estudado, um maior número de caso de gestantes com sífilis quando comparado a zona rural (Figura 4). De acordo com a literatura as pessoas da população urbana se relacionam sexualmente com um número maior de parceiros. Isto pode levar a um aumento na probabilidade de um indivíduo sem parceiro sexual estável ou fixo adquirir infecções sexualmente transmissíveis como a sífilis (BRASIL, 2011).

O maior número de casos confirmados foi nos testes NÃO TREP do que nos testes TREP. Isso provavelmente ocorreu porque os testes TREP são mais específicos localizando somente os anticorpos para a bactéria, enquanto os testes NÃO TREP localizam anticorpos específicos e não específicos para a bactéria Treponema pallidum (BRASIL, 2015).

Houve um alto número de casos confirmados de sífilis em gestantes por classificação primária. Supõe-se que após o primeiro sintoma, no caso o cancro duro, a paciente busca assistência hospitalar, quando recebe o diagnóstico de sífilis primaria (AVELLEIRA et al, 2006).

O maior número de óbito em gestantes com sífilis foi por outra causa. De acordo com a literatura mais da metade de mortes maternas no mundo foram por hemorragia, hipertensão e septsemia (SAY, 2014).

\section{CONCLUSÃO}

O maior número de exames possibilita um melhor tratamento e diminuição do número de casos. Entretanto, a ausência de cuidado preventivo durante o

$\mathrm{RC}: 66576$

Disponível em: https://www.nucleodoconhecimento.com.br/saude/sifilis-em-gestantes 
relacionamento sexual induz ao aumento de casos, principalmente entre mulheres em idade reprodutiva.

Áreas com maior população apresentam maiores números de casos. A variação alta de parceiros nestas áreas impulsiona o aumento de contaminação.

O tipo de teste influencia nos números. Testes menos específicos, mais gerais, localizam anticorpos específicos e não específicos para a bactéria. Isso pode alterar os números reais. O cuidado feminino com a saúde faz com quer o diagnóstico seja precoce, facilitando o tratamento.

A sífilis não é responsável direta pelo maior número de mortalidade em gestantes.

\section{REFERÊNCIAS}

AVELLEITA, J. C. R.; BOTTINO, G; Sífilis: Diagnóstico, Tratamento e Controle. An. Bras. Dermatol, Vol.81 no.2 Rio de Janeiro Mar./Apr. 2006.

BRASIL São Paulo, Centro de Referência e Treinamento DST/Aids. Boletim Epidemiológico. Ano XXX. 2013.

BRASIL, Ministério da Saúde. Secretaria de Ciência, Tecnologia e Insumos Estratégicos. Relatório de Recomendação, n¹59, 2015. Disponível em: <file://C:/Users/aluno.SELABI/Desktop/CURSO\%20DE\%20ESCRITA/(BRASIL,\%20 2015).pdf>. Acesso em:26/09/2017.

BRASIL. Ministério da Saúde Secretaria de Vigilância em Saúde - Departamento de DST, Aids e Hepatites Virais SAF SUL Trecho 2 Boletim Epidemiológico - Sífilis Ano IV- no 1, 2015.

BRASIL. Ministério da Saúde. Ministério da Saúde Lança Campanha de Combate à Sífilis. Disponível em: <http://www.brasil.gov.br/saude/2013/10/ministerio-dasaude-lanca-campanha-de-combate-a-sifilis>. Acesso em: 19/09/2017.

$\mathrm{RC}: 66576$

Disponível em: https://www.nucleodoconhecimento.com.br/saude/sifilis-em-gestantes 
BRASIL. Ministério da Saúde. Portal da Saúde. Combate á Sífilis Congênita. Disponivel em: <http://portalarquivos.saude.gov.br/campanhas/sifilis/>. Acesso em: 19/09/2017.

BRASIL. Ministério da Saúde. Secretaria de Vigilância em Saúde. Departamento de DST, Aids e Hepatites Virais. Pesquisa de conhecimento, atitudes e práticas na população brasileira / Ministério da Saúde. Secretaria de Vigilância em Saúde. Departamento de DST, Aids e Hepatites Virais. - Brasília: Ministério da Saúde, 2011.

BRASIL. Ministério da Saúde. Secretaria de Vigilância em Saúde. Departamento de DST, Aids e Hepatites Virais. Pesquisa de conhecimento, atitudes e práticas na população brasileira / Ministério da Saúde. Secretaria de Vigilância em Saúde. Departamento de DST, Aids e Hepatites Virais. - Brasília: Ministério da Saúde, 2011.

BRETAS, J. R. S.; OHARA, C. V. S.; JARDIM, D. P.; MUROYA, R. L.; Conhecimento Sobre DST/AIDS por Estudantes Adolescentes. Rev. esc. enferm. USP vol.43 no.3 São Paulo Sept. 2009.

CARVALHO, I. S.; BRITO, R. S; Sífilis congênita no Rio Grande do Norte: estudo descritivo do período 2007-2010. Epidemiol. Serv. Saúde, v.23 n.2 Brasília, 2014.

COSTA, C. C.; FEITAS, L. V.; SOUSA, D. M. N.; OLIVEIRA, L. L.; CHAGAS, A. C. M. A.; LOPES, M. V. O.; DAMASCENO, A. K. C. Sífilis Congênita No Ceará: Análise Epidemiológica De Uma Década. Rev Esc Enferm USP 2013; 47(1):149-56.

IBGE, Censo Demográfico 1960, 1970, 1980, 1991, 2000 e 2010. Disponível em: $<$ https://censo2010.ibge.gov.br/sinopse/index.php?dados=8>. Acesso em: 26/09/2017.

JUNIOR, W. B.; SHIRATSU, R.; PINTO, V.; Abordagem nas Doenças Sexualmente Transmissíveis. An Bras Dermatol. 2009;84(2):151-59.

RC: 66576

Disponível em: https://www.nucleodoconhecimento.com.br/saude/sifilis-em-gestantes 
KALININ, Y.; NETO, A. P.; PASSARELLI, D. H. C. Sífilis: aspectos clínicos, transmissão, Manifestações orais, diagnóstico e tratamento. Odonto 2015; 23(4546): 65-76.

MESQUITA, K.; LIMA, G. K.; FILGUEIRA, A.; FLÔR, S. M.; FREITAS, C. A.; LINHARES, M. S.; GUBERT, F.Análise dos Casos de Sífilis Congênita em Sobral, Ceará: Contribuições para Assistência Pré-Natal. DST - J bras Doenças Sex Transm 2012; 24(1):20-27

ROCHA, K.D., FECURY, A.A., OLIVEIRA, E., DENDASCK, C.V., DIAS, C.A.G.M. Number of congenital syphilis cases in Brazil between 2009 and 2013. Revista Científica Multidisciplinar Núcleo do Conhecimento. Year 05, Ed. 05, Vol. 01, pp. 131-143. May 2020. ISSN:2448-0959.

DOI:

10.32749/nucleodoconhecimento.com.br/health/congenital-syphilis-cases.

SAY, L.; CHAU, D.; GEMMILL, A.; TUBÇALP, O.; MOLLER, A.; DANIELS, J.; GULMEZOGLU, A. M.; TEMMERMAN, M.; ALKEMA, L. Global Causes Of Maternal Death: A Who Systematic Analysis. Lancet Glob Health, Vol.2, 2014.

SUTO, C. S. S.; SILVA, D. L.; ALMEIDA, E. S.; COSTA, E. L.; EVANGELISTA, T. J. Assistência Pré-Natal A Gestante Com Diagnóstico De Sífilis Revista de Enfermagem e Atenção à Saúde 2016; 5(2): 18-33.

Enviado: Novembro, 2020.

Aprovado: Novembro, 2020.

RC: 66576

Disponível em: https://www.nucleodoconhecimento.com.br/saude/sifilis-em-gestantes 